\title{
(C) OPEN ACCESS \\ Influence of social relationship domains and their combinations on incident dementia: a prospective cohort study
}

\author{
Tami Saito, ${ }^{1}$ Chiyoe Murata, ${ }^{1}$ Masashige Saito, ${ }^{2}$ Tokunori Takeda, ${ }^{3}$ Katsunori Kondo ${ }^{4,5}$
}

\begin{abstract}
- Additional material is published online only. To view please visit the journal online (http://dx.doi.org/10.1136/ jech-2017-209811).
\end{abstract}

${ }^{1}$ Department of Social Science, National Center for Geriatrics and Gerontology, Obu, Japan ${ }^{2}$ Faculty of Social Welfare, Nihon Fukushi University, Mihama,

Aichi, Japan

${ }^{3}$ Faculty of Rehabilitation and Care, Seijoh University, Tokai, Aichi, Japan

${ }^{4}$ Center for Preventive Medical Science, Chiba University, Chiba, Chiba, Japan

${ }^{5}$ Department of Gerontological Evaluation, National Center for Geriatrics and Gerontology, Obu, Japan

\section{Correspondence to} Dr Tami Saito, Department of Social Science, National Center for Geriatrics and Gerontology, Obu, Aichi 474-8511, Japan; t-saito@ncgg.go.jp

Received 2 August 2017 Revised 17 October 2017 Accepted 18 October 2017 Published Online First 31 October 2017

\begin{abstract}
Background Social relationships consist of mutually related but distinct dimensions. It remains unclear how these domains independently contribute to incident dementia. This large-scale, prospective cohort study examines associations between the social relationship domains as well as their combinations and incident dementia among community-dwelling older adults. Methods We analysed data from 13984 communitydwelling adults aged 65+ without long-term care needs living in Aichi prefecture in Japan. Incident dementia was assessed based on the Long-term Care Insurance records, followed for 3436 days from the baseline survey conducted in 2003. Three social relationships domains (social support, social networks and social activities) were further divided into a total of eight subdomains. A social relationship diversity score was calculated using the social relationship domains which were significantly related to incident dementia.
\end{abstract}

Results A Cox proportional hazards model showed that being married, exchanging support with family members, having contact with friends, participating in community groups and engaging in paid work were related to a lower likelihood of developing incident dementia, controlling for covariates and other social relationship domains. The diversity scores, ranging from 0 to 5 , were linearly associated with incident dementia $(p<0.001)$, and those who scored highest were $46 \%$ less likely to develop incident dementia compared with those in the lowest category.

Conclusions Our findings revealed five social relationship subdomains which were negatively related to incident dementia, suggesting that dementia may potentially be prevented by enhancing these social relationships. Future studies should examine independent pathways between each social relationship domain and incident dementia.

\section{INTRODUCTION}

Dementia is a major disease burden among older adults which threatens an individual's independence, imposes a serious caregiving burden on family caregivers and increases social care costs in the community. ${ }^{1}$ Because there is no established treatment to cure dementia, prevention is an important issue in ageing societies.

Social relationships and other modifiable risk/ protective factors such as lifestyle-related diseases, smoking and physical and leisure activities can also contribute to dementia prevention. ${ }^{2} 3$ However, further research is necessary to establish dementia prevention schemes, particularly those focusing on social relationship enrichment. ${ }^{2}$ Social relationships have been diversely defined; some studies only include interpersonal networks with others. In other studies, social relationships refer to connections between individuals and their social environment. ${ }^{4}$ The latter typically consist of several mutually related and distinct dimensions: a qualitative (or functional) dimension of social support and a quantitative (or structural) dimension of social integration, ${ }^{4}$ which include both social networks and engagement in social activities, such as participation in groups or paid/unpaid work. ${ }^{5}$ These social relationship domains can be further classified into subdimensions. For instance, social networks and support might be subdivided by the nature of the relationships, such as whether they involve family members, relatives or friends, ${ }^{6}$ while social activities might be subdivided based on where the activities take place, such as community-related or work-related activities. Social relationships are known to protect older adults against health problems by improving health behaviour (eg, smoking cessation), increasing psychological resources (eg, self-efficacy) and ameliorating physiological function (eg, immune system). ${ }^{7}$

Regarding incident dementia or cognitive decline, recent epidemiological studies have reported associations with social networks, ${ }^{8-10}$ social activities, ${ }^{911-14}$ and less consistently, social support. ${ }^{15}$ Other studies also showed that diverse social relationship engagement rather than dense participation in one specific social relationship can reduce the risk of cognitive decline or incident dementia. ${ }^{5}{ }^{16-19}$ However, each social relationship factor's independent contribution to incident dementia remains unclear despite the usefulness of this information for future intervention programmes or for motivating older adults to stay active in their communities. Most of the studies have not fully considered differences and similarities in the social relationship domains. To examine the relative importance of or independent pathways to incident dementia, it is important to compare the associations between each social relationship factor and incident dementia in the same analytic model. ${ }^{20-22}$

Several studies have succeeded in demonstrating associations between specific social relationship factors and cognitive decline, even after adjusting for other social relationship domains. ${ }^{6} 20 \quad 23-25$ These findings suggest the need for further longitudinal studies which use larger sample sizes, look 
at combinations of these potentially protective social relationships and include non-Western countries. Particularly in Asian countries where a rapid increase in the number of people with dementia has been observed, ${ }^{26}$ associations between social relationships and health could be different from those in Western countries. $^{11}$

In this study, we aim to examine the relationships between a variety of social relationship variables and their combinations and incident dementia in older Japanese adults.

\section{METHODS}

\section{Study sample}

Data were drawn from the Aichi Gerontological Evaluation Study prospective cohort, which is part of the Japan Gerontological Evaluation Study project. The primary aim of this ongoing project is to clarify the social determinants of health in community-dwelling older adults. A total of 33152 respondents who were 65 years or older, functionally independent and not eligible for Long-term Care Insurance (LTCI) benefits were selected using random sampling from six relatively large-scale municipalities and complete enumeration from four small-scale municipalities in Aichi prefecture, located in Central Japan. The self-administered questionnaire survey was conducted in October 2003 with a response rate of $52.1 \%$, which was higher than or comparable to that of other similar surveys. ${ }^{27}$ After excluding respondents who could not be identified in the LTCI records because of a lack of basic information, 15313 respondents were linked to the LTCI records dataset, which includes follow-up from the date of incident dementia for 3436 days $\mathbf{9 . 4}$ years) from 1 November 2003. The details of the survey are available elsewhere. ${ }^{28}$

In this study, respondents who had basic activities of daily living (BADL) disabilities or provided no BADL information at baseline $(n=1225)$ and those who experienced incident dementia within 1 year from the baseline $(n=104)$ were excluded to avoid the possibility of reverse causation that a possible preclinical cognitive decline in the majority of such respondents may lead to withdrawal from social relationships. ${ }^{10}$ The final sample for analysis consisted of data from 13984 respondents (see online supplementary figure S1).

\section{MEASUREMENTS}

\section{Outcome variable}

In this study, incident dementia was assessed based on the LTCI records. The Degree of Autonomy in the Daily Lives of Elderly Individuals with Dementia Scale, developed by the Ministry of Health and Welfare (now the Ministry of Health, Labour and Welfare of Japan), measures the degree of interference in one's ability to perform daily living activities because of symptoms, behaviours or communication difficulties caused by dementia on a scale which is scored as 0 or I to IV. ${ }^{29}$ This scale was validated by demonstrating its high correlation with the Mini Mental State Evaluation (MMSE; $r=-0.74)^{30}$ and its accordance with the Clinical Dementia Rating (CDR). ${ }^{31}$ Under the LTCI, assessments to determine the care-need levels of older adults were conducted through both home visit interviews by a trained healthcare professional and an examination by a primary physician, who almost always used the scale referenced above. In this study, we considered level II or above (a state in which a subject at least manifests some symptoms, behaviours or communication difficulties which may hinder daily activities) to indicate dementia, as described in previous studies. ${ }^{32} 33$

\section{Explanatory variables}

Social networks, social support and social activity variables were assessed as social relationship variables. We assessed marital status, contact with relatives and contact with friends as social network variables. All variables were dichotomised as present or absent. Marital status was dichotomised as married/bereaved, divorced, separated or never married. Social support variables were assessed based on answers to four questions: 'Do you have someone who looks after you when you are sick and confined to bed for a few days?' 'Do you look after someone when he/ she is sick and confined to a bed for a few days?' 'Do you have someone to listen to your concerns or complaints?' 'Do you listen to someone's concerns and complaints?' We assessed these items for each co-resident family member/relative/friend and neighbour and regarded the subjects as having some social support exchanges in each category if they answered 'yes' to at least one among the four questions. For older Japanese adults, co-residence with an adult child, often accompanied by the child's spouse and children, is not unusual ${ }^{34}$; therefore, familial support reflects support from both spouses and other co-resident family members. Social activity variables were assessed in terms of participating in any type of community group (eg, sports groups, local associations or other groups) and engagement in any kind of paid work. Having cohabitants (considered a social network indicator) was excluded from this study because it is highly correlated with social support exchange with co-resident family members $(r>0.6)$.

\section{Covariates}

We included diabetes, stroke, depression, subjective cognitive impairment (SCI), instrumental activities of daily living (IADLs), physical activity, leisure activity, education, household income, gender and age as possible confounders. ${ }^{13}{ }^{17}$ Diabetes and stroke were assessed by asking respondents if they had been diagnosed with these illnesses. Depression was assessed with a 15 -item version of the Geriatric Depression Scale (GDS) ${ }^{35}$ and dichotomised $(<5 / \geq 5)$. SCI was measured with one item which asked if the respondents often perceived themselves confusing times and places. As no standardised question for measuring SCI exists to the best of our knowledge, we conducted a preparatory analysis and confirmed a significant association between SCI and both incident dementia and social relationship factors. To consider other dimensions of early cognitive decline, we assessed IADLs, which are known to be impacted in the event of such a decline. ${ }^{36}$ IADLs were assessed with a five-item subscale from the Tokyo Metropolitan Institute of Gerontology Higher Competence Scale, for which validity and reliability were confirmed. ${ }^{37}$ Scores were dichotomised and respondents were grouped based on whether they had any difficulty on at least one item. As we did not assess physical activities directly, habitual walking ${ }^{38}$ was assessed instead and considered to be engaged in if they walked $30 \mathrm{~min}$ or longer per day. Leisure activity was assessed by asking if they had any hobbies. Years of education $(<10 / \geq 10)$, equivalised household income (low/middle/high), gender (male/female) and age (65-69, 70-74, 75-79, 80-84 and $\geq 85$ years) were also assessed. Although studies have shown that tobacco consumption could elevate the risk of dementia, ${ }^{2}{ }^{3}$ we did not examine this due to a high correlation with gender $(r>0.6)$.

\section{Analysis}

All variables included missing data except for outcome, age and gender. The highest rate of missing data was $17.8 \%$ observed for household income, and a total of $45.2 \%$ of the analysed 
data had a missing score for at least one study variable. Therefore, we performed a multiple imputation by chained equations, assuming the data were missing at random. ${ }^{39}$ We generated 20 datasets, analysed them separately and pooled the estimates and SEs applying Rubin's rules. ${ }^{40}$ Respondents who were lost in follow-up due to death or relocation before incident dementia were censored.

Using the imputed data, a Cox proportional hazards model was employed to examine the association between social relationship variables and incident dementia. In model 1, we examined a crude HR for each social relationship variable, followed by model 2, which examined an HR for each social relationship variable controlling for covariates. In model 3 , all social relationship variables were entered simultaneously with all covariates. Following these models, we examined the cumulative risk reduction for incident dementia by engaging in diverse social relationships. We generated a social relationship engagement score by adding the number of social relationship variables in which respondents were engaged. The variables were selected according to the significance of their relationships with the outcome in model 3. Both trend test and departure from linear trend were examined. Additionally, we conducted a series of sensitivity analyses: we examined the effect modification by gender, GDS, SCI and IADLs to confirm whether the association between a social relationship variable and incident dementia differed by gender or by the presence of health risks which could result in withdrawal from social relationships. Similarly, we conducted analyses excluding respondents who developed incident dementia within 2 or 3 years, respectively, to confirm whether such reverse causality exists. We confirmed in advance that correlation coefficients among social relationship variables and covariates did not exceed 0.4 and checked the proportional hazard assumption, first graphically and then by checking Schoenfeld residuals. The significance level was set at $\mathrm{p}<0.05$ in all analyses using SPSS V.22.0J.

\section{RESULTS}

The average follow-up days were 2878.3, with 2131 cases (15.2\%) developing dementia during the follow-up period. Table 1 shows the characteristics of the respondents; the mean age was 72.6 , and $50.9 \%$ were women. Over $80 \%$ of the respondents were independent with their IADLs.

Table 2 shows the association between social relationship variables and incident dementia. In model 1 , all eight social relationship variables were significantly related to incident dementia, with an $8 \%-50 \%$ risk reduction among respondents having each of these social relationships. In model 2, which controlled for covariates, the HR for all social relationship variables was closer to 1.0. In model 3, controlling for other social relationship variables in addition to the covariates, respondents who had contact with friends and/or were married, participating in community groups, engaging in paid work and exchanging support with their family members were $11 \%-17 \%$ less likely to develop incident dementia. The inter-relationships among social relationship variables (see online supplementary table S1) partially explained the significant association between contact with relatives and incident dementia. Relationships between covariates and outcomes in model 3 are shown in online supplementary table S2.

Finally, we produced a cumulative score of these five variables $(y e s=1 ;$ no $=0$ ) to examine the association between diverse social relationship engagement and incident dementia. Cronbach's alpha for these five items was 0.40 . We found that respondents who scored $2(n=2460), 3(n=3833), 4(n=4980)$

\begin{tabular}{|c|c|c|c|}
\hline Variables & Categories & $\mathrm{N}(\%)$ & $\%$ onset \\
\hline \multirow[t]{5}{*}{ Age } & $65-69$ & $5097(36.4)$ & 5.0 \\
\hline & $70-74$ & $4182(29.9)$ & 11.9 \\
\hline & $75-79$ & $2850(20.4)$ & 23.6 \\
\hline & $80-84$ & $1302(9.3)$ & 35.3 \\
\hline & $\geq 85$ & $553(4.0)$ & 44.7 \\
\hline \multirow[t]{2}{*}{ Gender } & Male & $6860(49.1)$ & 13.3 \\
\hline & Female & $7124(50.9)$ & 17.1 \\
\hline \multirow[t]{2}{*}{ Education } & $<10$ & $8326(59.5)$ & 16.5 \\
\hline & $\geq 10$ & $5658(40.5)$ & 13.3 \\
\hline \multirow[t]{3}{*}{ Household income } & Low & $5848(41.8)$ & 16.9 \\
\hline & Middle & $6444(46.1)$ & 13.7 \\
\hline & High & $1693(12.1)$ & 15.6 \\
\hline \multirow[t]{2}{*}{ Geriatric Depression Scale } & $<5$ & $9833(70.3)$ & 13.0 \\
\hline & $\geq 5$ & $4151(29.7)$ & 20.5 \\
\hline \multirow[t]{2}{*}{ Subjective cognitive complaint } & Yes & $2072(14.8)$ & 26.2 \\
\hline & No & $11913(85.2)$ & 13.3 \\
\hline \multirow[t]{2}{*}{ Instrumental activities of daily living } & Independent & $11268(80.6)$ & 12.8 \\
\hline & Dependent & $2716(19.4)$ & 25.2 \\
\hline \multirow[t]{2}{*}{ Stroke } & Yes & $232(1.7)$ & 25.7 \\
\hline & No & $13752(98.3)$ & 15.1 \\
\hline \multirow[t]{2}{*}{ Diabetes } & Yes & $1616(11.6)$ & 18.1 \\
\hline & No & $12368(88.4)$ & 14.9 \\
\hline \multirow[t]{2}{*}{ Daily walking time (min) } & $\geq 30$ & 9040 (64.6) & 14.4 \\
\hline & $<30$ & 4945 (35.4) & 16.8 \\
\hline \multirow[t]{2}{*}{ Hobby } & Yes & $10762(77.0)$ & 13.4 \\
\hline & No & $3222(23.0)$ & 21.4 \\
\hline
\end{tabular}

Imputed data are used.

or $5(\mathrm{n}=1818)$ were $14 \%, 25 \%, 35 \%$ or $46 \%$ less likely to develop incident dementia compared with those who scored 0 or 1 ( $n=893)$, respectively, controlling for the covariates. A significant dose-response relationship was also observed with regard to the association between the combined score and incident dementia (trend $\mathrm{p}<001$; figure 1). Baseline characteristics according to the cumulative score groups are shown in online supplementary table $\mathrm{S} 3$.

Additionally, we observed almost no effect modification by gender, GDS, SCI or IADLs, except for an interaction of being married by gender $(p=0.001)$, indicating that the association between having a spouse and incident dementia was stronger in men (see online supplementary table S4). Even when we excluded the cases of incident dementia within 2 and 3 years from the analysis, the results were, in general, similar to our initial analysis, which excluded respondents with dementia onset within 1 year. However, the risk reduction by being married (HR 0.96, $95 \% \mathrm{CI} 0.85$ to 1.08 ), group participation (HR $0.91,95 \% \mathrm{CI}$ 0.82 to 1.02 ) and work engagement (HR $0.91,95 \%$ CI 0.80 to 1.04) shrank when we excluded the respondents with $\leq 3$-year dementia incidence (see online supplementary table S5).

\section{DISCUSSION}

We examined the association between multiple social relationship domains and incident dementia using data from community-dwelling, healthy older adults. The findings of this study showed that being married, having support exchanges with co-resident family members, having contact with friends, 
Table 2 Cox proportional hazards model estimating the association between social relationship variables and incident dementia

\begin{tabular}{|c|c|c|c|c|}
\hline Variables & Categories & Model $1 *$ & Model $2^{\dagger}$ & Model 3‡ \\
\hline & $(n, \%)$ & HR $(95 \% \mathrm{Cl})$ & $\mathrm{HR}(95 \% \mathrm{Cl})$ & $\mathrm{HR}(95 \% \mathrm{Cl})$ \\
\hline Married & Yes $(10232,73.2)$ & 0.50 (0.46 to 0.54$)$ & 0.85 (0.76 to 0.94$)$ & 0.88 (0.79 to 0.99$)$ \\
\hline Contact (relatives) & Yes $(12990,92.9)$ & 0.74 (0.64 to 0.87 ) & 0.85 (0.73 to 1.00$)$ & $0.89(0.76$ to 1.05$)$ \\
\hline Contact (friends) & Yes $(12291,87.9)$ & 0.55 (0.49 to 0.62 ) & 0.78 (0.69 to 0.88 ) & $0.83(0.73$ to 0.94$)$ \\
\hline Group participation & Yes $(8671,62.0)$ & 0.61 (0.56 to 0.67 ) & 0.85 (0.77 to 0.94$)$ & 0.89 (0.80 to 0.98$)$ \\
\hline Work engagement & Yes $(3480,24.9)$ & 0.52 (0.47 to 0.59$)$ & 0.85 (0.76 to 0.97 ) & 0.88 (0.77 to 0.99$)$ \\
\hline Support (family) & Yes $(11507,82.3)$ & 0.68 (0.62 to 0.75$)$ & 0.84 (0.76 to 0.94 ) & $0.88(0.79$ to 0.99$)$ \\
\hline Support (relatives) & Yes $(7372,52.7)$ & 0.92 (0.84 to 1.00$)$ & 1.02 (0.93 to 1.11$)$ & $1.03(0.95$ to 1.13$)$ \\
\hline Support (friends) & Yes $(6121,43.8)$ & 0.75 (0.69 to 0.82 ) & 0.96 (0.87 to 1.05$)$ & 0.98 (0.89 to 1.08$)$ \\
\hline
\end{tabular}

*Unadjusted model.

†Association with each social variable and incident dementia is estimated. All covariates (age, gender, education, household income, Geriatric Depression Scale, subjective cognitive complaints, instrumental activities of daily living, stroke, diabetes, daily walking time and hobbies) are adjusted.

$\ddagger$ All social variables are simultaneously entered into the model with all covariates.

participating in community groups and engaging in paid work were negatively related to incident dementia. This study supports the findings of a meta-analytic study based on 19 longitudinal studies $^{22}$ in relation to social activity participation and social networks. Furthermore, our findings suggest that social relationship factors may have distinct mechanisms in incident dementia or cognitive decline. ${ }^{172124}$

Although mechanisms which intervene between social relationship factors and incident dementia remain unclear, several pathways could exist. First, cognitive stimulation through social relationship engagement may delay incident dementia. ${ }^{17}$ Engagement in jobs or group activities which require high cognitive skills could reduce the risk of incident dementia. ${ }^{14}$ Effective communication with others could also stimulate cognition through recalling shared experiences or solving problems. ${ }^{24}$ This engagement may also help older adults maintain their cognitive abilities even with the presence of severe disease pathology (ie, cognitive reserve hypothesis). ${ }^{17}$

The second possible pathway is that rich social relationships may enhance a healthy lifestyle through easier access to health

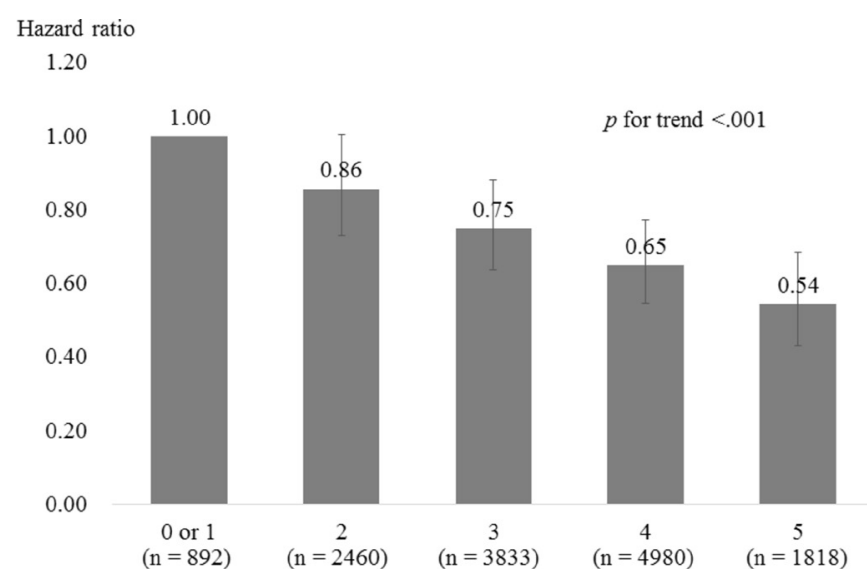

Figure 1 Hazard risks for incident dementia by diverse social relationship engagement score. The scores are calculated by adding the number of selected social relationship variables in which respondents engaged. The five social relationship variables are selected according to model 3: married, contact with friends, group participation, work engagement and social support exchanges with family. All covariates (age, gender, education, household income, Geriatric Depression Scale, subjective cognitive complaints, instrumental activities of daily living, stroke, diabetes, daily walking time and hobbies) are adjusted. information and more social control to restrict undesirable health-related behaviours. ${ }^{7}$ In particular, the presence of a spouse is a well-known resource for health because of the social support and control over health behaviours. ${ }^{41}$ Because the findings of this study show a significant association between the presence of a spouse and incident dementia, even when adjusting for social support, it is possible that marital control may work to restrict undesirable health behaviours.

Third, effective stress management through rich social relationships may mitigate harmful stress nervous system responses. ${ }^{1620}$ Social support is a well-known buffer against the negative effects of stress on health. ${ }^{4}$ In this study, relationships with family had a significant association with incident dementia, which is supported by the findings from Asian ${ }^{15}$ as well as Mediterranean countries. ${ }^{23}$ This suggests the possible importance of familial support, particularly in countries where a familism tradition exists.

However, unfortunately, these possibilities remain hypothetical because we could not demonstrate which pathways exist between social relationship factors and incident dementia. Further studies should examine the detailed pathways to incident dementia by considering physiological indicators, accessibility to health information and factors reflecting cognitive stimulation.

Additionally, this study shows that the aggregated score of the social relationship factors had a negative and linear relationship with incident dementia, which could also support the theory that each social relationship factor made an independent contribution to incident dementia. In this study, people with the highest social relationship diversity score were $46 \%$ less likely to develop onset dementia compared with those with the lowest score. Several studies also showed similar findings for a protective association between diverse social relationships and incident dementia, ${ }^{17} 18$ with a 37\%-57\% risk reduction, although they did not measure identical social relationship variables and outcomes. These findings suggest that diverse social relationship engagement could be a promising intervention scheme for the prevention of dementia, similar to other more established modifiable risk factors such as diabetes mellitus, midlife hypertension, smoking or physical inactivity. ${ }^{3}$

We demonstrated a negative association between several social relationship domains and incident dementia by comparing them in an identical dataset. Our study sample exceeded 13000 cases, which was another advantage. Most studies on the association between social relationships and cognitive decline used much smaller samples, which could 
be a reason for inconsistency in the findings. In addition, we showed the cumulative advantage of diverse social relationship engagement in preventing dementia.

At the same time, we must discuss several limitations to this study. First, we assessed incident dementia using the LTCI dataset, rather than a clinical diagnosis of dementia. This might cause misclassification to some degree. However, the LTCI dementia scale criteria is validated in accordance with the scores of the MMSE ${ }^{30}$ or the CDR. ${ }^{31}$ The scale is also nationally standardised and rarely suffers from self-response bias. Second, we assessed social relationship variables and covariates only at the baseline. Therefore, we could neither examine changes in social relationship engagement levels over time nor confirm causal relationships between social relationship factors and covariates. Our study used a sample of healthy older adults; we excluded those with BADL disabilities at baseline and those who developed incident dementia within a year; we controlled for multiple health dimensions and other covariates; and we confirmed no statistical effect modification by the health covariates. However, we cannot deny the possibility of reverse causation. For instance, our sensitivity analysis revealed that the HRs for group participation and work engagement were similar but slightly closer to 1.0 than those in our initial analysis when we excluded respondents with early dementia onset. Further studies should replicate the findings of this study using an intervention design or, at the very least, using long-term panel data with multiple survey times. Third, it is possible that our measurement of social relationship variables is not enough to comprehensively assess the social relationships of individuals. For instance, the qualitative nature of social support (eg, positive/negative dimensions), which we did not assess but which could be related to health, ${ }^{42}$ may be worth examining in future studies.

In conclusion, this study revealed five social relationship subdomains which were negatively related to incident dementia using a large-scale prospective cohort study. Additional analysis showed that respondents engaging in these five

\section{What is already known on this subject}

- Studies suggest that social relationships can potentially protect against cognitive decline or incident dementia.

- Specific dimensions of social relationships, such as social networks, social activity participation or social support, were shown to be negatively related to incident dementia; however, a majority of studies did not consider their mutual relationships in the analysis.

\section{What this study adds}

- This study reveals the five social relationship subdomains which were related to incident dementia: being married, having support exchanges with co-resident family members, making contact with friends, participating in community groups and engaging in paid work.

- This study also shows that engaging in diverse social relationships were less likely to develop incident dementia by almost $50 \%$.

- These findings suggest the potential for enhancing social relationships to prevent dementia in older adults who desire social relationships, particularly for those lacking familial ties. domains was less likely to develop dementia by almost $50 \%$. Adequate support programmes to enhance social networks with friends and neighbours and participation in social activities could potentially prevent dementia in older adults who desire social relationships but do not have such opportunities, particularly unmarried adults or those lacking familial ties.

Acknowledgements The authors thank Professor Kenta Murotani for his statistical advice and the JAGES group members for their helpful suggestions.

Contributors All authors contributed to the conception and design of this study. Data collection was primarily conducted by KK. Analyses were performed by TS and supported by CM and MS. TS prepared the initial manuscript and CM, MS, TT and KK significantly contributed to revising it. All authors read and approved the final manuscript.

Funding This study was supported by the MEXT-Supported Program for the Strategic Research Foundation at Private Universities (2009-2013), Grant-in-Aid for Scientific Research (KAKENHI) (23243070, 18390200, 25713027 and 16KT0014) and grants (24-17 and 27-18) from the National Center for Gerontology and Geriatrics.

\section{Competing interests None declared.}

Ethics approval The study protocol and informed consent procedure were approved by the Nihon Fukushi University Ethics Committee. The study was conducted in compliance with the Declaration of Helsinki.

Provenance and peer review Not commissioned; externally peer reviewed.

Open Access This is an Open Access article distributed in accordance with the Creative Commons Attribution Non Commercial (CC BY-NC 4.0) license, which permits others to distribute, remix, adapt, build upon this work non-commercially, and license their derivative works on different terms, provided the original work is properly cited and the use is non-commercial. See: http://creativecommons.org/ licenses/by-nc/4.0/

(c) Article author(s) (or their employer(s) unless otherwise stated in the text of the article) 2018. All rights reserved. No commercial use is permitted unless otherwise expressly granted.

\section{REFERENCES}

1 Prince MJ, Wu F, Guo Y, et al. The burden of disease in older people and implications for health policy and practice. Lancet 2015;385:549-62.

2 Flicker L. Modifiable lifestyle risk factors for Alzheimer's disease. J Alzheimers Dis 2010;20:803-11.

3 Norton S, Matthews FE, Barnes DE, et al. Potential for primary prevention of Alzheimer's disease: an analysis of population-based data. Lancet Neurol 2014;13:788-94

4 Cohen S. Social relationships and health. Am Psychol 2004;59:676-84.

5 Hughes TF, Flatt JD, Fu B, et al. Engagement in social activities and progression from mild to severe cognitive impairment: the MYHAT study. Int Psychogeriatr 2013;25:587-95.

6 Zunzunegui MV, Alvarado BE, Del Ser T, et al. Social networks, social integration, and social engagement determine cognitive decline in community-dwelling Spanish older adults. J Gerontol B Psychol Sci Soc Sci 2003;58:S93-S100.

7 Berkman LF, Glass T, Brissette I, et al. From social integration to health: Durkheim in the new millennium. Soc Sci Med 2000;51:843-57.

8 Barnes DE, Cauley JA, Lui LY, et al. Women who maintain optimal cognitive function into old age. J Am Geriatr Soc 2007:55:259-64.

9 Lee $\mathrm{SH}, \mathrm{Kim}$ YB. Which type of social activities may reduce cognitive decline in the elderly?: a longitudinal population-based study. BMC Geriatr 2016;16:165.

10 Sörman DE, Rönnlund M, Sundström A, et al. Social relationships and risk of dementia: a population-based study. Int Psychogeriatr 2015;27:1391-9.

11 Glei DA, Landau DA, Goldman N, et al. Participating in social activities helps preserve cognitive function: an analysis of a longitudinal, population-based study of the elderly. Int J Epidemiol 2005;34:864-71.

12 James BD, Wilson RS, Barnes LL, et al. Late-life social activity and cognitive decline in old age. J Int Neuropsychol Soc 2011;17:998-1005.

13 Marioni RE, Proust-Lima C, Amieva H, et al. Social activity, cognitive decline and dementia risk: a 20-year prospective cohort study. BMC Public Health 2015;15:1089.

14 Then FS, Luck T, Luppa $M$, et al. Differential effects of enriched environment at work on cognitive decline in old age. Neurology 2015;84:2169-76.

15 Zhu S, Hu J, Efird JT. Role of social support in cognitive function among elders. J Clin Nurs 2012;21:2118-25.

16 Bassuk SS, Glass TA, Berkman LF. Social disengagement and incident cognitive decline in community-dwelling elderly persons. Ann Intern Med 1999;131:165-73.

17 Fratiglioni L, Wang HX, Ericsson K, et al. Influence of social network on occurrence of dementia: a community-based longitudinal study. Lancet 2000;355:1315-9. 
18 Saczynski JS, Pfeifer LA, Masaki K, et al. The effect of social engagement on incident dementia: the Honolulu-Asia Aging Study. Am J Epidemiol 2006;163:433-40.

19 Stoykova R, Matharan F, Dartigues JF, et al. Impact of social network on cognitive performances and age-related cognitive decline across a 20-year follow-up. Int Psychogeriatr 2011;23:1405-12.

20 Eisele M, Zimmermann T, Köhler M, et al. Influence of social support on cognitive change and mortality in old age: results from the prospective multicentre cohort study AgeCoDe. BMC Geriatr 2012:12:9.

21 Gow AJ, Corley J, Starr JM, et al. Which social network or support factors are associated with cognitive abilities in old age? Gerontology 2013;59:454-63.

22 Kuiper JS, Zuidersma M, Oude Voshaar RC, et al. Social relationships and risk of dementia: a systematic review and meta-analysis of longitudinal cohort studies. Ageing Res Rev 2015;22:39-57.

23 Béland F, Zunzunegui MV, Alvarado B, et al. Trajectories of cognitive decline and social relations. J Gerontol B Psychol Sci Soc Sci 2005;60:P320-P330.

24 Glymour MM, Weuve J, Fay ME, et al. Social ties and cognitive recovery after stroke: does social integration promote cognitive resilience? Neuroepidemiology 2008;31:10-20

25 Seeman TE, Lusignolo TM, Albert M, et al. Social relationships, social support, and patterns of cognitive aging in healthy, high-functioning older adults: MacArthur studies of successful aging. Health Psychol 2001;20:243-55.

26 World Health Organization. Dementia: a public health priority: World Health Organization, 2012.

27 Fujiwara T, Kondo K, Shirai K, et al. Associations of childhood socioeconomic status and adulthood height with functional limitations among Japanese older people: results from the JAGES 2010 Project. J Gerontol A Biol Sci Med Sci 2014;69:852-9.

28 Nishi A, Kondo K, Hirai H, et al. Cohort profile: the ages 2003 cohort study in Aichi, Japan. J Epidemiol 2011;21:151-7.

29 Ministry of Health, Labour and Welfare. The degree of autonomy in the daily lives of elderly individuals with dementia scale. http://www.mhlw.go.jp/topics/2013/02/dl/ tp0215-11-11d.pdf (accessed 19 July 2017).
30 Hisano S. The relationship between Revised Hasegawa Dementia Scale (HDS-R) Mini-Mental State Examination (MMSE) and Bed-fast Scale, dementia scale. Japanese J Geriatr Psychiatry 2009.

31 Meguro K, Tanaka N, Kasai M, et al. Prevalence of dementia and dementing diseases in the old-old population in Japan: the kurihara project. Implications for long-term care insurance data. Psychogeriatrics 2012;12:226-34.

32 Murata C, Takeda T, Suzuki K, et al. Positive affect and incident dementia among the old. J Epidemiol Res 2015;2.

33 Tomata Y, Sugiyama K, Kaiho Y, et al. Green tea consumption and the risk of incident dementia in elderly Japanese: the Ohsaki Cohort 2006 study. Am J Geriatr Psychiatry 2016:24:881-9.

34 Takagi E, Silverstein M, Crimmins E. Intergenerational coresidence of older adults in Japan: conditions for cultural plasticity. J Gerontol B Psycho/ Sci Soc Sci 2007;62:S330-S339.

35 Yesavage JA, Brink TL, Rose TL, et al. Development and validation of a geriatric depression screening scale: a preliminary report. J Psychiatr Res 1982;17:37-49.

36 Jutten RJ, Peeters CFW, Leijdesdorff SMJ, et al. Detecting functional decline from normal aging to dementia: development and validation of a short version of the amsterdam IADL questionnaire. Alzheimers Dement 2017:8:26-35.

37 Koyano W, Shibata H, Nakazato K, et al. Measurement of competence: reliability and validity of the TMIG index of competence. Arch Gerontol Geriatr 1991;13:103-16.

38 Wong CH, Wong SF, Pang WS, et al. Habitual walking and its correlation to better physical function: implications for prevention of physical disability in older persons. J Gerontol A Biol Sci Med Sci 2003:58:M555-M560.

39 Schafer JL, Graham JW. Missing data: our view of the state of the art. Psychol Methods 2002:7:147-77.

40 Rubin DB. Multiple Imputation after 18+ Years. J Am Stat Assoc 1996;91:473-89.

41 Joutsenniemi K, Martelin T, Martikainen P, et al. Living arrangements and mental health in Finland. J Epidemiol Community Health 2006;60:468-75.

42 Stafford M, McMunn A, Zaninotto P, et al. Positive and negative exchanges in social relationships as predictors of depression: evidence from the English longitudinal study of aging. J Aging Health 2011:23:607-28. 